LOUIS CARTON (1861-1924)

AND THE RELIGIONS OF AFriCA

\title{
Louis Carton (1861-1924) et les religions d'Afrique
}

Jean-Louis Podvin

Université Littoral Côte d' Opale, UR 4030 HLLI

jean-louis.podvin@univ-littoral.fr - https://orcid.org/0000-0002-9957-026X

Fecha recepción 31/03/2020 | Fecha aceptación 26/10/2021

\section{Abstract}

As a medical officer, Louis Carton discovered Tunisia in 1886 and devoted himself with passion to archaeology. He discovered numerous sites and became a reference in this field, even if he quickly encountered difficulties with the official archaeological authorities. Even though he was more specialized in Roman colonisation and irrigation works, he often touched on religious matters.

\section{Keywords}

African Saturn, Bulla Regia, Dougga, Louis Carton, primitive Christianity.

\section{Résumé}

Médecin militaire, Louis Carton découvre la Tunisie en 1886 et s'adonne avec passion à l'archéologie. Il y découvre de nombreux sites, et devient une référence dans ce domaine, même s'il entre rapidement en lutte avec les autorités archéologiques officielles. Plus spécialisé dans les domaines de la colonisation romaine et des travaux d' irrigation, il aborde cependant à de multiples reprises le champ religieux.

\section{Mots-clés}

Bulla Regia, christianisme primitif, Dougga, Louis Carton, Saturne africain. 

Né À Saint-Omer (Pas-de-Calais, France) en 1861, Louis Benjamin Charles Carton y commence ses études, puis rejoint la région de Lille (Nord, France) quand ses parents y déménagent ${ }^{1}$. Après le baccalauréat, il entame des études de médecine et embrasse, peu après ses 20 ans, une carrière militaire en tant qu'élève à l'hôpital militaire lillois ${ }^{2}$. Sorti en janvier 1884 de l'École d'application du Val de Grâce à Paris, il est d'abord affecté au chef-lieu de son département d'origine, à Arras, où il s'ennuie d'après ses propres écrits, avant de rejoindre la brigade d'occupation de Tunisie en octobre 1886 et de découvrir, très rapidement, les immenses richesses archéologiques du pays. Il exhume et met en valeur de nouveaux sites archéologiques puniques et romains, comme Bulla Regia et Thuburnica, et profite en cela de différents postes qu'il assure successivement ${ }^{3}$. Les très nombreuses publications qu'il réalise à partir de 1888 sont autant de jalons qui éclairent des lieux qu'il a fréquentés.

Louis Carton avait un certain nombre de thèmes de prédilection. Il s'est ainsi beaucoup intéressé aux questions écologiques, au climat et à l'environnement antiques, et à l'utilisation de l'eau dans l'espace nord-africain, sans doute en relation avec les objectifs colonialistes de la France dans cet espace nouveau pour elle ${ }^{4}$. Carton ne fut donc pas un spécialiste de la religion. Cependant, ses multiples découvertes lui permirent de mettre au jour des témoignages religieux qu'il fit connaître par ses très nombreux ouvrages, articles et conférences. Dans le

1. J.-L. Podvin, «Louis Carton et sa région natale : un attachement indéfectible », dans J.-L. Podvin (éd.), Louis Carton, de Saint-Omer à Tunis, Aachen, 2017, 11-32.

2. Cl. Fredj, « Louis Carton, médecin militaire en Tunisie », dans J.-L. Podvin (éd.), Louis Carton..., op. cit., n. 1, 45-61. Il est $5^{\mathrm{e}}$ au concours d'admission au service de santé militaire : Le Mémorial artésien, 25 octobre 1882 .

3. R. Hanoune, "Louis Carton, archéologue : notice biographique ", dans C. Demeulenaere-Douyère (éd.), Explorations et voyages scientifiques de l’Antiquité à nos jours, Paris, 2008, 573-580 ; J.-P. Laporte, «Un archéologue en Tunisie, Louis Carton (1861-1924) ", Bulletin Archéologique du Comité des Travaux Historiques et Scientifiques, 35, 2009, 239-264.

4. N. Kallala, «Dr Louis Carton, un archéologue d'époque coloniale, ou de l'archéologie appliquée à l'agriculture », Africa, 22, 2008, 229-245. 
cadre de cette étude, notre propos vise à mettre en évidence les incursions que Louis Carton a effectuées dans le domaine religieux au fil de ses publications ${ }^{5}$.

Le $\mathrm{D}^{\mathrm{r}}$ Carton - c'est ainsi qu'il signe la plupart de ses publications - se révèle très vite un découvreur hors pair de sites archéologiques en Tunisie, un territoire jusqu'alors peu exploité par les archéologues. En 1889-1890, il fouille à proximité de Souk el-Arba où il est cantonné, et il exhume plusieurs centaines de sépultures à Bulla Regia : une partie du mobilier funéraire orna même les vitrines de l'Exposition universelle à Paris. Il publie cette même année une brève note de trois pages ${ }^{6}$ sur une stèle découverte à Sidi Mohamed-el-Azreg, faisant référence à un temple (aedes) dédié au grand dieu africain, Saturne. Il est ensuite affecté à Téboursouk où il révèle, à proximité, le site de Thugga (Dougga). Dans le récit de sa vie qu'il donne en $1911^{7}$, il raconte comment il y découvrit le temple de Saturne : il avait repéré une pierre inscrite, et s'était attelé à en comprendre le sens ; il en sortit d'autres de terre qu'il retourna pour déchiffrer leurs inscriptions, et il retrouva ainsi la dédicace du temple de Saturne. Avec l'aide du lieutenant Denis, en poste à Téboursouk, puis de l'ordonnance E. Obrecht, il réalisa plusieurs sondages dans l'espace ainsi découvert et obtient le soutien de l'Académie des Inscriptions et Belles-Lettres, puis du Ministère de l'Instruction publique. Il envoie les stèles qu'il exhume au musée Alaoui à Tunis. C'est sa première véritable incursion dans le domaine religieux.

Dès 1891, il présente plus en détail la découverte du temple de Saturne à Dougga ${ }^{8}$, un édifice de $53 \mathrm{~m}$ sur $28 \mathrm{~m}$ daté de la dernière décennie du $\mathrm{II}^{\mathrm{e}}$ siècle, situé sur un promontoire dominant la ville, et dont il décrit les éléments de la dédicace et les principales parties : portique antérieur (terrasse et pronaos), partie moyenne (grande cour péristyle sur trois de ses côtés), partie postérieure (trois cellae), et citernes souterraines. Il évoque également les fouilles menées à El-Matria (Civitas Nimlulitana) où il dégage un petit temple de $15 \mathrm{~m}$ sur $8 \mathrm{~m}$, dédié sous Marc Aurèle à Jupiter Optimus Maximus, Juno Regina et Minerva Augusta. Il revient à plusieurs reprises sur ce monument élevé par un particulier, C. Memmius Pecuarius, et le décrit avec précision?.

À Thuburnica, il découvre un temple dont il croit reconnaître les cellae et qu'il pense pouvoir attribuer à Junon ou au Génie de la colonie d'après les fragments d'inscriptions

5. Nous avons dressé la liste de ses publications : «Bibliographie de Louis Carton », dans J.-L. Podvin (éd.), Louis Carton..., op. cit., n. 1, 171-182.

6. L. Carton, «Un temple de Saturne en Tunisie », Comptes Rendus des Séances de l'Académie des Inscriptions et Belles-Lettres, 1890, 466-468.

7. L. Carton, «Manifestation en l'honneur de M. le docteur Carton», Revue Tunisienne, 1911, 193-194.

8. L. Carton, «Rapport sur les fouilles exécutées en 1891 », Comptes Rendus des Séances de l'Académie des Inscriptions et Belles-Lettres, 1891, 437-449. Il y revient dans sa " Notice sur les fouilles exécutées à Dougga (Tunisie) », Bulletin de la Société de Géographie et d'Archéologie de la Province d'Oran, 13, 1893, 63-82, où il détaille aussi une série de stèles dédiées à Saturne et Tanit retrouvées sur place.

9. L. Carton avec le lt. Denis, "Numluli et son capitole ", Bulletin Archéologique du Comité des Travaux Historiques et Scientifiques, 1893, 74-80 ; "Municipium Numlulitanum », Revue Tunisienne, 7, 1900, 395410 et Revue Tunisienne, 8, 1901, 79-102. 
relevés in situ, et un autre sanctuaire dédié à Mercure, de l'époque de Caracalla. Il publie aussi d'autres inscriptions de Thuburnica en l'honneur de Vénus, Mars, Priape, Concordia, Luna, Sol et du genius Sesase (?) ${ }^{10}$. Plus tard, il exhume, à l'emplacement de la ferme qu'il y a achetée, un sanctuaire de $17 \mathrm{~m}$ sur $16 \mathrm{~m}$ qu'il attribue à Saturne compte tenu des similitudes avec celui des fouilles d'El-Kénissia et des artefacts qu'il y a trouvés (vases gravés au nom du dieu, stèles votives romaines, grecques et néo-puniques) ${ }^{11}$ : doté de trois cellae, il est précédé d'un portique entourant une cour ; c'est à l'extérieur que furent découverts les objets votifs, mêlés à des cendres.

Dans son article de la même année sur la région de Souk el-Arba ${ }^{12}$, il énumère nombre de constructions, notamment hydrauliques (bassins, réservoirs, aqueducs, puits), agricoles (bâtiments, pressoirs) et défensives (forteresses et fortins) qu'il a rencontrées, et il évoque à plusieurs reprises la possibilité de l'existence de temples en se fondant notamment sur la présence de chapiteaux de colonnes (Henchir ed-Dekkir) ou d'une cella (temple ou porte triomphale à Aïn-Cherchar). Dans cette étude, il n'oublie évidemment pas Bulla Regia, le site qui l'avait fait connaître.

En 1895, son monumental article (plus de 400 pages) sur la région de Dougga fait date $^{13}$. Il revient sur les deux monuments connus à Dougga jusqu’à ses découvertes - le temple de Jupiter et le mausolée libyco-punique - et propose un cheminement systématique le long les voies romaines du secteur. Il examine les sites où il a pu identifier des ruines romaines et les décrit. Outre les monuments, il apporte des éléments épigraphiques mentionnant, ici le génie d'Agbia (p. 46) ou un flamine perpétuel (pp. 49, 106), là un monument à Saturne (pp. 70-71, 102-104, 112, 131-132, 145-146) ou à Victoria (p. 126). À Dougga, il mentionne le sanctuaire de Neptune et ses environs (pp. 159-170), celui de Saturne (p. 178), mais aussi les églises (pp. 171-172). À Henchir ez-Zaouïa, il évoque un temple d'Esculape (pp. 200-204); à El-Bouïa, un temple indéterminé (pp. 221-225) ; à Henchir Brahim Riah, une basilique chrétienne (p. 231); à Sidi Abdallah Melliti, une église «byzantine" (pp. 281-284); à Henchir Redès, une église (pp. 291-294); à Numluli, une autre église quadrifoliée et le Capitole (pp. 294-300) ; à Henchir oued-Soussa, un temple de Fortuna (pp. 242-243); à Sustri, un possible sanctuaire à Saturne (pp. 317-319); à Uchi Maius, une dédicace à Saturne (p. 257) et une autre d'un temple à Cérès (pp. 276-277).

10. L. Carton (avec M. Chenel), "Thuburnica», Bulletin Archéologique du Comité des Travaux Historiques et Scientifiques, 1891, 161-192. On consultera aussi R. Hanoune, "Carton et Thuburnica », dans J.-L. Podvin (éd.), Louis Carton..., op. cit., n. 1, 63-74.

11. L. Carton, "Note sur la découverte d'un sanctuaire de Saturne dans la 'Colonia Thuburnica' ", Comptes Rendus des Séances de l'Académie des Inscriptions et Belles-Lettres, 1907, 380-384; " Note sur des fouilles exécutées à Thuburnica et à Chemtou ", Bulletin Archéologique du Comité des Travaux Historiques et Scientifiques, 1908, 410-444.

12. L. Carton, «Essai de topographie archéologique sur la région de Souk-el-Arba », Bulletin Archéologique du Comité des Travaux Historiques et Scientifiques, 1891, 207-247.

13. L. Carton, "Découvertes épigraphiques et archéologiques faites en Tunisie (région de Dougga) ", Mémoires de la Société des Sciences, de l'Agriculture et des Arts de Lille, 1895, 1-427. 
En 1897, il publie une autre étude sur le sanctuaire de Saturne à Dougga ${ }^{14}$. Il revient sur le sujet dans la Revue tunisienne l'année suivante ${ }^{15}$, et critique le nom de « Temple de Baal-Saturne » qui a été donné au sanctuaire restitué au cours d'une exposition de 1898 : il propose de garder le nom de Baal pour un premier état, mais de prendre celui de Saturne pour un second, de considérer six colonnes en façade et non quatre, et il revient sur divers ornements du temple, critiquant avec virulence nombre de propositions de M. Parmentier, en lui reprochant surtout de ne pas l'avoir consulté avant de réaliser ses planches en couleur... Carton se montre imbu de lui-même, cela lui causera de multiples ennuis avec les autorités archéologiques qui se mettent en place.

On retrouve Carton à Dougga à propos d'un temple situé près de l'hippodrome, au fond d'une cour de $49 \mathrm{~m}$ sur $34 \mathrm{~m}^{16}$. Précédé d'un pronaos dont ne subsistait qu'un chapiteau corinthien, il comportait une cella terminée par une abside (Fig. 11.1), le tout accessible par un escalier. Selon lui, ce temple était dédié à Eschmoun, puisque deux autres du même type l'étaient déjà à Baal-Saturne et Tanit-Célestis.

À propos de sépultures à enceinte qui faisaient l'objet d'offrandes, il estime qu'elles dépassent la simple tombe, et se présentent comme des sanctuaires, avec aire et enclos ${ }^{17}$.

Le découvreur s'intéresse aussi à de petits objets. Ainsi, il publie des artéfacts trouvés à Philippeville, en un lieu considéré comme un sanctuaire de Mithra, et entrés ensuite dans la collection Théry à Lille ${ }^{18}$ : il connaît bien la capitale des Flandres pour y avoir vécu et aussi parce qu'il y a été muté depuis 1891, sans que cela ne l'empêche de retourner régulièrement en Tunisie pour des campagnes de fouilles. Il publie notamment une tête de statue juvénile coiffée du bonnet phrygien.

En 1902, il se penche sur des statuettes de Vénus et une d'Attis (?) découvertes, avec des lampes, dans des sépultures d'Hadrumète ${ }^{19}$. Il étudie aussi des statues de plus grande taille comme celles, en terre cuite, trouvées à Hammam-Lif ${ }^{20}$, et en profite pour aborder la question des cachettes. S’il n'est pas sûr que les panthères réparties symétriquement à un canthare sur un bas-relief de la région de Thuburnica soient vraiment bacchiques comme

14. L. Carton, «Le sanctuaire de Baal Saturne à Dougga, rapport sur les fouilles exécutées à Dougga en 1893 », Nouvelles Archives des Missions, 7, 1897, 367-474.

15. L. Carton, « Le temple de Saturne (de Dougga) à l'Exposition nationale des Beaux-Arts de 1898 ", Revue Tunisienne, 5, 1898, 307-314.

16. L. Carton, «Un édifice de Dougga en forme de temple phénicien », Mémoires de la Société Nationale des Antiquaires de France, 56, 1897, 52-60.

17. L. Carton, «Les sépultures à enceinte de la Tunisie », L’Anthropologie, 8, 1897, 27-40.

18. L. Carton, "Objets trouvés dans un sanctuaire de Mithra à Constantine », Bulletin de la Société Nationale des Antiquaires de France, 1899, 327-330.

19. L. Carton, "Statuettes en terre cuite de la nécropole d'Hadrumète ", Mémoires de la Société Nationale des Antiquaires de France, 61, 1902, 230-243.

20. L. Carton, "Statues en terre cuite de grandeur naturelle trouvées à Hammam-Lif (Tunisie) », Bulletin de l'Académie d'Hippone, 32, 1912-1913, 23-30. 
il l'avance ${ }^{21}$, il a toutefois remarqué que les artistes avaient vraisemblablement puisé à la source de monuments dionysiaques.

En 1899, en partant de l'ouvrage de R. Cagnat et P. Gauckler sur Les Monuments historiques de la Tunisie paru l'année précédente, le médecin archéologue développe un long propos sur l'architecture sacrée polythéiste en Afrique ${ }^{22}$. Il loue la qualité de la publication, qui a d'abord permis aux auteurs de mettre en évidence les sanctuaires et les autels dont il a été possible de déterminer à qui ils étaient consacrés (30 temples dont on connaît l'emplacement et l'attribution, 103 dont on ne connait que l'attribution), puis les indéterminés (47 dont on devine la destination), et enfin ceux dont on peut penser qu' ils étaient des temples (16 édifices). Il revient ensuite sur les temples « païens » en Afrique. Les Capitoles (7 temples) sont en tête, comme celui, prostyle et tétrastyle de Dougga, mais aussi ceux d'Henchir es-Souar, flanqué de deux cellae dans le pronaos, d'Henchir Matria, de Medeina et de Sbeitla. Les temples d'Apollon seul ou avec Diane sont ensuite mentionnés, puis ceux de Caelestis (10 sanctuaires dont 8 temples), de Cérès ( 9 sanctuaires dont 8 temples), de Concordia, d'Esculape (8 temples), d'Hercule (4 sanctuaires dont 2 temples), de Junon, de Jupiter, des Lares, de Liber Pater (6 sanctuaires dont 5 temples), de Mars, de Mater Magna (Mactar), de Mercure (11 sanctuaires dont 10 temples), de Neptune, de Pluton (7 sanctuaires dont 4 temples), et bien entendu de Saturne (19 temples). Pour ce dernier, il peut s'agir d'un espace à ciel ouvert (Djebel Bou Korneïn) ou d'un temple au plan particulier (Dougga). Des inscriptions suggèrent l'existence d'autres sanctuaires. Carton s'interroge sur certaines attributions, comme à Aïn-Tounga (temple de Caelestis ou temple des eaux ?) et El-Bouia (temple ou mausolée ?). Il se demande comment interpréter certaines cryptes retrouvées dans le sous-sol de plusieurs temples. L'auteur date de l'époque antonine les plus importants chantiers, même si, dans certains cas, ils sont plus tardifs (première moitié du $\mathrm{III}^{\mathrm{e}} \mathrm{s}$. pour Victoria). Leur situation est variable, mais les sanctuaires de Saturne sont plutôt situés sur des hauteurs, ceux du dieu guérisseur Esculape près de sources d'eau ${ }^{23}$. La plupart, tétrastyles, sont tournés vers l'Est ; ceux abritant plusieurs divinités sont pourvus de niches ad hoc, ou de plusieurs cellae. Rares sont ceux en gros appareil, car on a privilégié des pratiques anciennes, à base de blocage revêtu d'un enduit, ce qui n'est pas sans conséquence sur la pérennité des édifices. Le fait que des particuliers, des associations, ou de petites communautés n’hésitaient pas à élever des sanctuaires explique peut-être les moyens réduits déployés. Carton détaille un grand nombre d'exemples de ces donateurs, et rapporte aussi les sommes engagées dans ces constructions, allant de 125 sesterces pour des marches, à 150000 sesterces pour le temple de Saturne à Dougga! Sauf rares exceptions comme le temple central du Capitole à Sbeitla, les temples étaient dépourvus d'ouvertures pour l'éclairage.

21. L. Carton, « Panthères bacchiques affrontées sur un bas-relief de l'Afrique du Nord », Revue Archéologique, 1902, 332-335.

22. L. Carton, "L'architecture sacrée de l'Afrique païenne d'après un livre de MM. Cagnat et Gauckler », Bulletin de la Société de Géographie et d'Archéologie de la Province d'Oran, 19, 1899, 133-167.

23. Il en apporte un exemple concret, avec un bâtiment bien conservé au bordj d'Abd el-Melek, dans L. Carton, «Le Djebel Gorra », Revue Tunisienne, 8, 1901, 269-278. 
Esprit curieux, Carton s'interroge sur une forme sculptée sur un rocher à Bulla Regia, qu'il juge d'époque punique ${ }^{24}$. Il publie des stèles de Sidi Bou Rouis dont une porte non seulement la représentation de trois divinités mais aussi leur nom : Sol, Jupiter, Luna, en plus de celui de Fortuna. Une autre représente les Dioscures et Hercule ${ }^{25}$. Il en arrive à des considérations sur la position des divinités sur la stèle. Ses multiples publications sur des nécropoles (Hadrumète notamment) lui font là encore toucher les aspects religieux.

Il s'intéresse à quelques reprises à des thèmes chrétiens, comme un vase décoré d'une scène de martyre ${ }^{26}$. Il y revient dans d'autres articles consacrés aux lampes, qui concernent aussi bien des thématiques chrétiennes que polythéistes ${ }^{27}$. Il aborde aussi la question des églises chrétiennes, et " l'affaire d'Uppenna » qui oppose Carton aux autorités archéologiques représentées par Gauckler fait couler beaucoup d'encre ${ }^{28}$. Cet édifice donatiste, plusieurs fois remanié, avait à ses côtés un baptistère au sol couvert de mosaïque. Différents éléments font référence aux martyrs d'Uppenna, dans le contexte délicat en France de la séparation des Églises et de l'État.

Il fouille le temple punique de Tanit à El-Kénissia et en tire là encore un gros article ${ }^{29}$. Ce sanctuaire, situé à mi-pente comme c'est le cas à Dougga, est limité par une enceinte rectangulaire de $46 \mathrm{~m}$ sur $28 \mathrm{~m}$. Un portique à colonnes englobait une cour, et plusieurs bâtiments dont il reste peu de chose (Fig. 11.2). Plusieurs bases (d’autels ?) précédaient le grand escalier. Diverses pièces au-delà de cet escalier avaient le sol couvert de mosaïques, alors que les autres en étaient dépourvues. Des stèles en grande quantité, des offrandes calcinées (bœufs, moutons) mais aussi des statuettes (30), des lampes (au moins 3100) des brûle-parfum (1036) et des unguentaria (1423) y ont été dégagés. Carton examine les quelque 200 stèles, avec leurs symboles comme les palmettes, simples ou doubles ; la représentation stylisée

24. L. Carton, "Sculpture sur un rocher de Bulla Regia », Bulletin de la Société de Géographie et d'Archéologie de la Province d'Oran, 22, 1902, 237-240.

25. L. Carton, "Les stèles de Sidi Bou Rouis », Bulletin de la Société Archéologique de Sousse, 3, 1905, 201-211.

26. L. Carton, « Note sur un vase chrétien à reliefs figurés trouvé à Thélepte (Tunisie) en 1909 », Comptes Rendus des Séances de l'Académie des Inscriptions et Belles-Lettres, 1909, 597-605.

27. L. Carton, "L'art indigène sur les lampes de la Colonia Thuburnica », Mémoires de la Société Nationale des Antiquaires de France, 73, 1914, 141-168. Certaines de ses publications sur les lampes à huile demeurent toujours indispensables, comme nous avons pu le constater dans notre étude sur les lampes à décor isiaque en Afrique du Nord.

28. L. Carton, «Les basiliques chrétiennes d'Upenna », Bulletin de la Société Archéologique de Sousse, 4, 1906, 62-72. Sur ce sujet controversé, cf. D. Raynal, Archéologie et histoire de l'Église d'Afrique. Uppenna I-II, Toulouse, 2006 ; J.-M. Paillier, « Carton, Uppenna et Gauckler. Enjeux et coulisses d'un affrontement », dans J.-L. Podvin (éd.), Louis Carton..., op. cit., n. 1, 75-97.

29. L. Carton, «Le sanctuaire de Tanit à El-Kénissia ", Mémoires de l'Académie des Inscriptions et BellesLettres, 12.1, 1906, 1-160. Il a aussi publié une étude plus succincte sur El-Kénissia, englobant d'autres monuments comme le théâtre : Les fouilles d'El Kenissia (près Sousse), Paris, 1906, puis « Notice sur les ruines d’El-Kénissia (près Sousse) », Bulletin de la Société Archéologique de Sousse, 5, 1907, 68-93. 
du fidèle sous la forme d'un triangle pour le corps, d'un cercle pour la tête; des animaux et un gâteau ; un croissant renversé. D’autres portent l'emblème triangulaire de Tanit ( $n^{\circ} 38$ p. 43 à $\mathrm{n}^{\circ} 70$ p. 51), un cône ( $\mathrm{n}^{\circ} 71$ p. 51 à n ${ }^{\circ} 87$ p. 62), la fourche ou le gril $\left(\mathrm{n}^{\circ} 88\right.$ p. 62 à $\mathrm{n}^{\circ} 92$ p. 65), un rectangle ( $n^{\circ} 93$ p. 65 à $n^{\circ} 103$ p. 66), des symboles sexuels ( ${ }^{\circ} 104$ p. 67 à $n^{\circ} 113$ p. 71), ou encore un socle à gorge égyptienne ( $\mathrm{n}^{\circ} 114-115$ p. 71-74), un disque $\left(\mathrm{n}^{\circ} 116-121\right.$ p. 74 76 ), un croissant, des colonnes ( $\mathrm{n}^{\circ} 122-131$ p. 76-78), des personnages $\left(\mathrm{n}^{\circ} 131-139\right.$ p. $\left.78-82\right)$, un olivier, caducée, des animaux ( $\mathrm{n}^{\circ} 140-148$ p. 82-87). Les inscriptions sont rares (quatre inscriptions puniques seulement sur des stèles). L'auteur développe des considérations sur les stèles des autres sanctuaires à proximité. Il étudie aussi les lampes en terre cuite, d'abord puniques, puis romaines (pp. 96-105), et les brûle-parfums (pp. 105-106), les unguentaria, les «burettes ", les vases à ossements, ce qui lui permet de préciser le type d'animaux utilisés lors des sacrifices, en l'occurrence surtout des moutons. Les monnaies en bronze retrouvées in situ vont du milieu du II ${ }^{e}$ siècle av. J.-C. au $\mathrm{III}^{e}$ s. apr. J.-C. Trois statuettes complètes et d'autres fragmentaires sont détaillées et comparées avec d'autres exemplaires régionaux (pp. 117-133). Divers éléments retrouvés parmi les cendres sont également mentionnés. Toutes ces informations lui permettent de tirer quelques conclusions sur les rites religieux observés dans ce sanctuaire. Carton termine son étude en comparant le sanctuaire d'El-Kénissia avec d'autres, punico-romains : à ciel ouvert pour les uns, à plan sémitique pour d'autres ${ }^{30}$, à plan de temple romain pour les derniers. Il remarque que, dans le premier groupe, certains ont des chambres «sacrées " prolongeant le portique ou des cellae au centre de la cour. Le type d'ElKénissia lui permet de proposer de reconsidérer des sites jusqu'alors non identifiés comme des sanctuaires : à Hadrumète, sous l'église (pp. 141-148), mais aussi à Nora, en Sardaigne (pp. 149-152) ${ }^{31}$. Il termine sur les divinités honorées, Saturne, Tanit et Baal, et propose une restitution des rites et de la vie qui pouvait animer ledit sanctuaire ${ }^{32}$.

À Ghardimaou, il publie des stèles et une ruine qu'il considère être un sanctuaire de $6,75 \mathrm{~m}$ sur 2,75 m. La cella de 2,75 m sur $2 \mathrm{~m}$ est précédée par une cour ${ }^{33}$, et elle comportait des dalles formant deux niches qui abritaient peut-être des statues ou statuettes en terre cuite, retrouvées en morceaux à proximité. Une, mieux conservée, présente une tête de lion, que Carton considère être le Genius Terrae Africae, et qui n'est pas si éloignée de la déesse égyptienne Sekhmet. Plusieurs stèles en grès ont également été trouvées, les unes inscrites, les

30. Pour une remise en perspective des idées de Carton dans son époque, voir M. Sebaï, « La construction d'un mythe contemporain : les temples "sémitiques" d'Afrique romaine ", Anabases, 11, 2010, 265-179.

31. Il avait, l'année précédente, publié «Les nuraghes de Sardaigne », Bulletin de la Société Archéologique de Sousse, 3, 1905, 278-280.

32. Pour une vision plus récente, voir par exemple B. D’Andrea, «Il tofet di El Kénissia e il rapporto tra tofet tardo punici, santuari a Saturno e "paesaggi del potere" ", L'Africa romana, 19, 2012, 2479-2496.

33. L. Carton, « Note sur des édicules renfermant des statues en terre cuite découvertes dans la région de Ghardimaou (Tunisie) », Comptes Rendus des Séances de l'Académie des Inscriptions et Belles-Lettres, 1918, 338-347. 
autres anépigraphes (Fig. 11.3). Il revient sur des stèles dans l'étude d'une collection privée, en présentant notamment un exemplaire en marbre, relevé à El-Djem (Fig. 11.4) ${ }^{34}$.

À Bulla Regia en 1913-1914, il dégage de nouvelles inscriptions dans la zone des thermes $^{35}$. Il présente aussi l'église du prêtre Alexander, réalisée à partir de pierres réutilisées de bâtiments antérieurs, et qui comportait deux sacristies. Lélément qui avait incité Carton à fouiller à cet endroit était la présence de grosses pierres dépassant du sol. Il revient sur cette église devant l'Académie des Inscriptions et Belles-Lettres : le bâtiment présente une nef de plus de $5 \mathrm{~m}$ sur $4 \mathrm{~m}$, flanquée de deux bas-côtés de 2,6 $\mathrm{m}$, dont elle est séparée par des sortes d'auges ${ }^{36}$. Le fond de la nef était occupé par un presbyterium rectangulaire, à l'avant duquel a été retrouvé un reliquaire en plomb. Deux sacristies s'ouvraient de part et d'autre du presbyterium. L'ensemble a été ravagé par un incendie, dans les cendres duquel on a relevé des calices en verre et de gros récipients en terre cuite ayant contenu du blé, des pois chiches, des haricots, des amandes, des pêches, des cerises, des olives et des liquides. Parmi les objets en bronze retrouvés dans la sacristie méridionale, outre les amphores ${ }^{37}$, on a relevé un plat, un vase et trois croix dont une au nom du prêtre Alexander, de l'époque byzantine. Cette pièce était probablement le diaconicum, destiné aux objets rituels et sacrés, alors que le prothesis, au nord, était davantage destiné aux provisions de l'église. On notera que certains éléments de cette église avaient été récupérés de bâtiments antérieurs et de la nécropole toute proche. Carton s'intéresse tout particulièrement aux auges qui séparent la nef des bas-côtés, et suit Gsell dans son interprétation en faveur d'aménagements pour la préparation de repas, que saint Augustin avait tenté de combattre en Afrique ; les auges auraient aussi pu servir à récolter les offrandes des fidèles, avant qu'elles ne soient stockées dans les grands récipients des sacristies. Louis Carton approfondit sa pensée dans un autre article (Fig. 11.5), où il apporte une série de réalisations similaires en Afrique du Nord, comme pour la basilique de Tebessa ${ }^{38}$.

En 1922, peu avant sa mort, il signale encore la découverte de deux temples à Bulla Regia, à proximité des grands thermes publics.

Dans les dernières années de sa vie, Louis Carton se concentre sur Carthage ${ }^{39}$. Il se penche sur les aspects puniques de la ville, puis sur les quartiers romains. Plus qu'un découvreur,

34. L. Carton, « Note sur quelques collections privées de Sousse », Bulletin de la Société Archéologique de Sousse, 7, 1909, 119-122.

35. L. Carton, « Découvertes faites en 1914 dans les fouilles de Bulla Regia », Bulletin Archéologique du Comité des Travaux Historiques et Scientifiques, 1915, 184-208.

36. L. Carton, "L'église du prêtre Alexander découverte à Bulla Regia en 1914 », Comptes Rendus des Séances de l'Académie des Inscriptions et Belles-Lettres, 1915, 116-130.

37. Sur le contentieux entre Carton et l'administration à propos des poteries de l'église d'Alexander, cf. R. Hanoune, «Louis Carton, d'après quelques documents d'archives », dans J.-L. Podvin (éd.), Louis Carton..., op. cit., n. 1, 108.

38. L. Carton, «Les lignes d'auges des églises et des autres monuments de l'Afrique ancienne », Recueil de Notices et Mémoires de la Société Archéologique de Constantine, 49, 1915, 81-92.

39. L. Carton, «La Carthage punique d'après les récentes découvertes ", Revue Archéologique, 1923, 329338. 
il se fait surtout le défenseur des ruines de Carthage auprès d'un large public, que ce soit par le truchement d'ouvrages de vulgarisation ${ }^{40}$ ou de conférences ${ }^{41}$ qu'il organise aussi bien en Tunisie qu'en France (Paris, Lyon, Bordeaux, Saint-Omer, Alger) et même à l'étranger (Belgique par exemple), en s'appuyant habilement sur le Comité des dames amies de Carthage ${ }^{42}$. Une publication posthume rassemble même ses découvertes puniques autour de la gare de Salammbô ${ }^{43}$, recouvertes par la suite par une nécropole romaine.

Ses incursions dans le domaine religieux sont diverses et concernent aussi bien la période des polythéismes, qu'ils soient puniques ou romains, que celle du monothéisme chrétien, précoce et vigoureux en Afrique.

Certaines interprétations de Carton, très largement tributaires de son époque, ont, fort logiquement, pu être remises en cause par les dé veloppements plus récents de l'archéologie. Il n’en demeure pas moins que le travail effectué fut conséquent, notamment en ce qui concerne la protection de sites et la volonté de promouvoir une activité économique, en l'occurrence touristique, autour de l'archéologie ${ }^{44}$. Il a aussi su motiver d'autres fouilleurs ${ }^{45}$, et les plans qu' il a dressés ou les relevés qu'il a réalisés sont parfois les seuls encore disponibles à l'heure actuelle ${ }^{46}$.

40. L. Carton, La beauté des ruines de Carthage, Paris, 1923 ; Pour visiter Carthage, Tunis, 1924 ; Carthage inconnue, Tunis, 1924.

41. A. Mezzolani-Andreose, "Louis Carton, polémiste et conférencier au service de la Carthage antique ", dans J.-L. Podvin (éd.), Louis Carton..., op. cit., n. 1, 113-134.

42. H. Dridi et A. Mezzolani, «De Carthage à Neuchâtel en passant par le canton de Vaud. Quelques aspects de l'activité du Comité des Dames Amies de Carthage ", dans F. Briquel Chatonnet, C. Fauveaud et I. Gajda (éds.), Entre Carthage et l'Arabie heureuse. Mélanges offerts à François Bron, Paris, 2013, 317-331.

43. L. Carton, Sanctuaire punique découvert à Carthage, Paris, 1929.

44. À ce sujet, Cl. Gutron, "Voyager dans le temps avec un archéologue à travers la Tunisie coloniale : Louis Carton (1861-1924) et sa Tunisie en l'an 2000 », dans C. Demeulenaere-Douyère (éd.), Explorations et voyages scientifiques de l'Antiquité à nos jours, Paris, 2008, 553-571; M. Bacha, «Un archéologue amateur. Louis Carton (1861-1924) et le projet de parc archéologique de Carthage (Tunisie) », dans J.-Y. Andrieux et P. Harismendy (éds.), Initiateurs et entrepreneurs culturels du tourisme (1850-1950), Rennes, 2011, 21-33.

45. Voir par exemple J.-P. Laporte, "François Icard, un disciple et émule de Louis Carton », dans J.-L. Podvin (éd.), Louis Carton..., op. cit., n. 1, 135-170.

46. R. Hanoune, "Carton et Thuburnica», op. cit., n. 10, 64, le précise à propos du site de Thuburnica, situé dans une zone militaire interdite à la frontière avec l'Algérie. 
Monógrafico | Louis Carton (1861-1924) et les religions d'Afrique

\section{Figures}

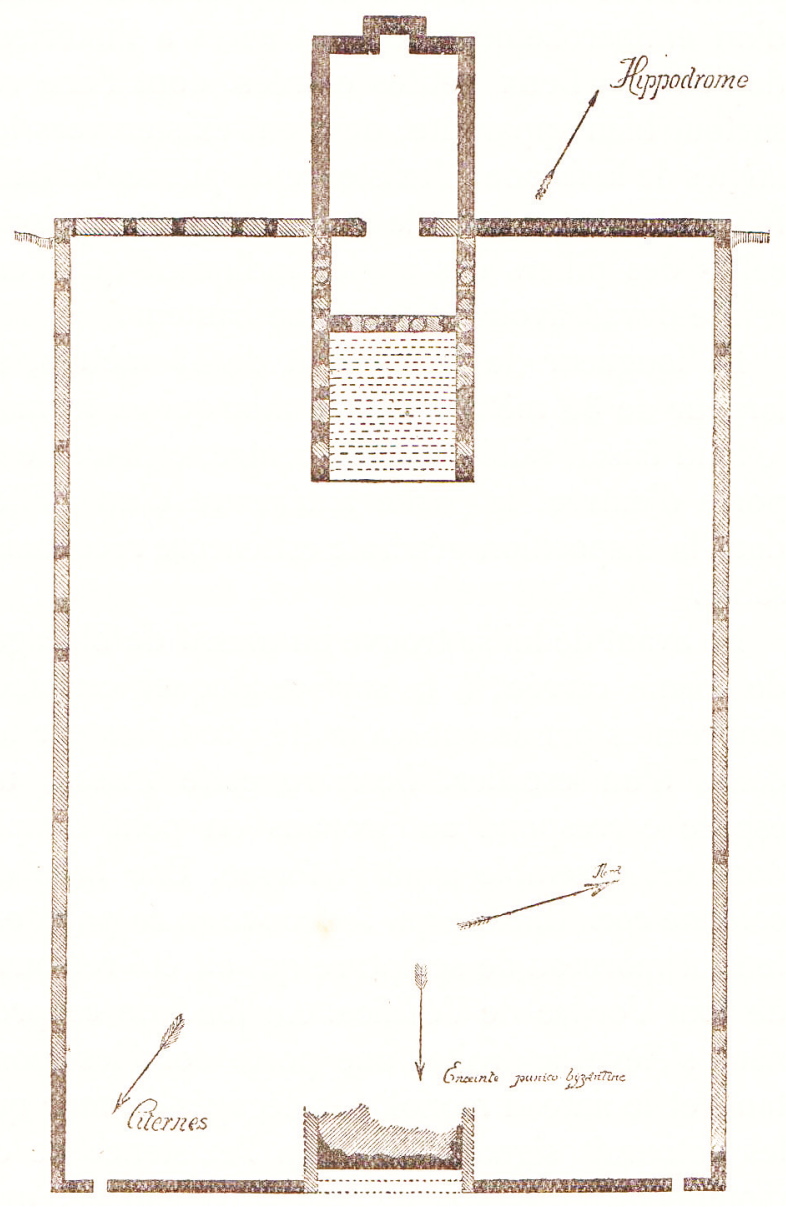

Fig. 1 : Plan d'un temple de Dougga (d'après L. Carton, « Un édifice de Dougga ...", op. cit., n. 16, 54). 


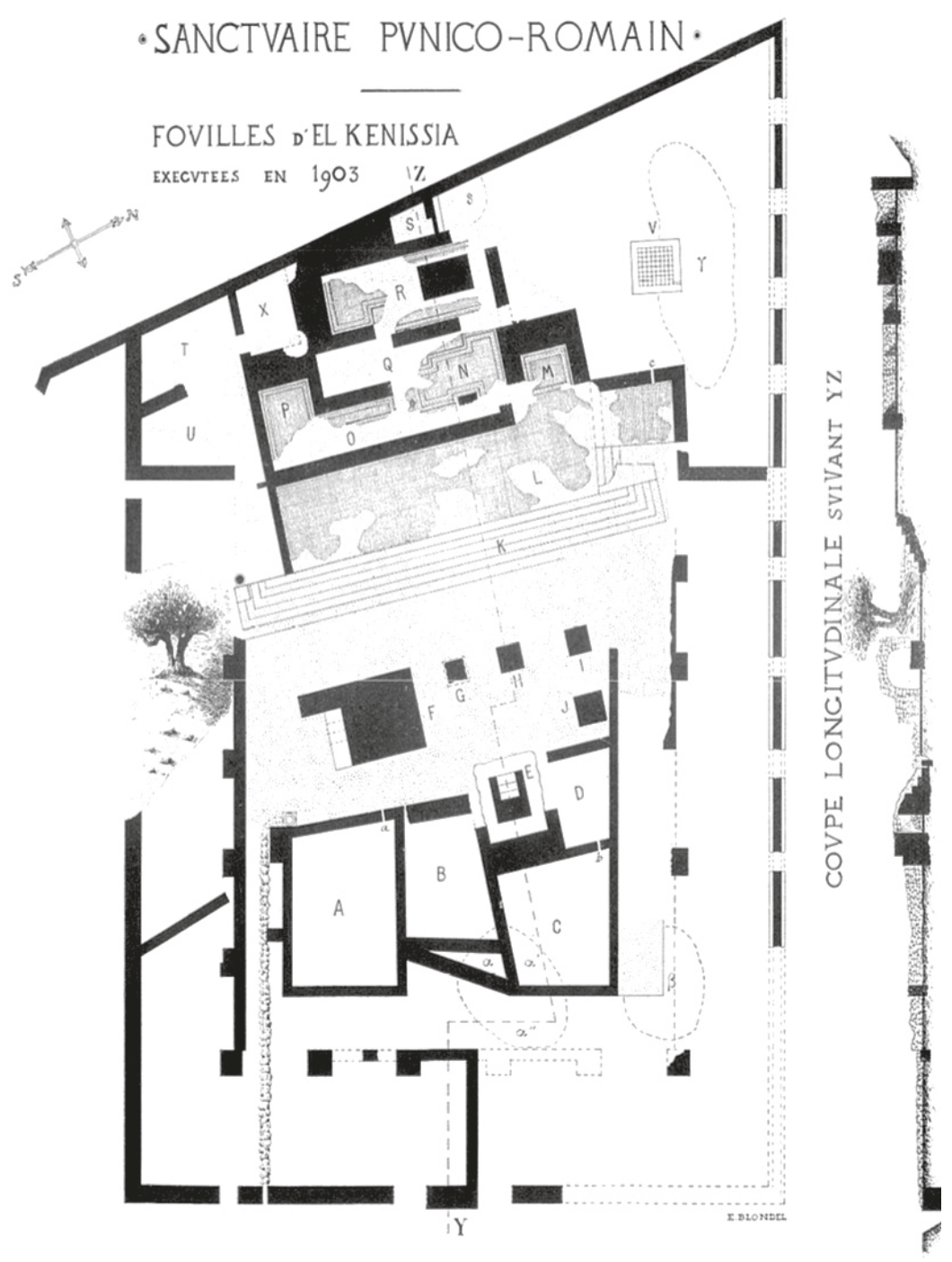

Fig. 2 : Sanctuaire punico-romain d'El-Kénissia (d'après L. Carton, « Le sanctuaire de Tanit à El-Kénissia ", op. cit., n. 29, pl. I). 
Monógrafico | Louis Carton (1861-1924) et les religions d'Afrique

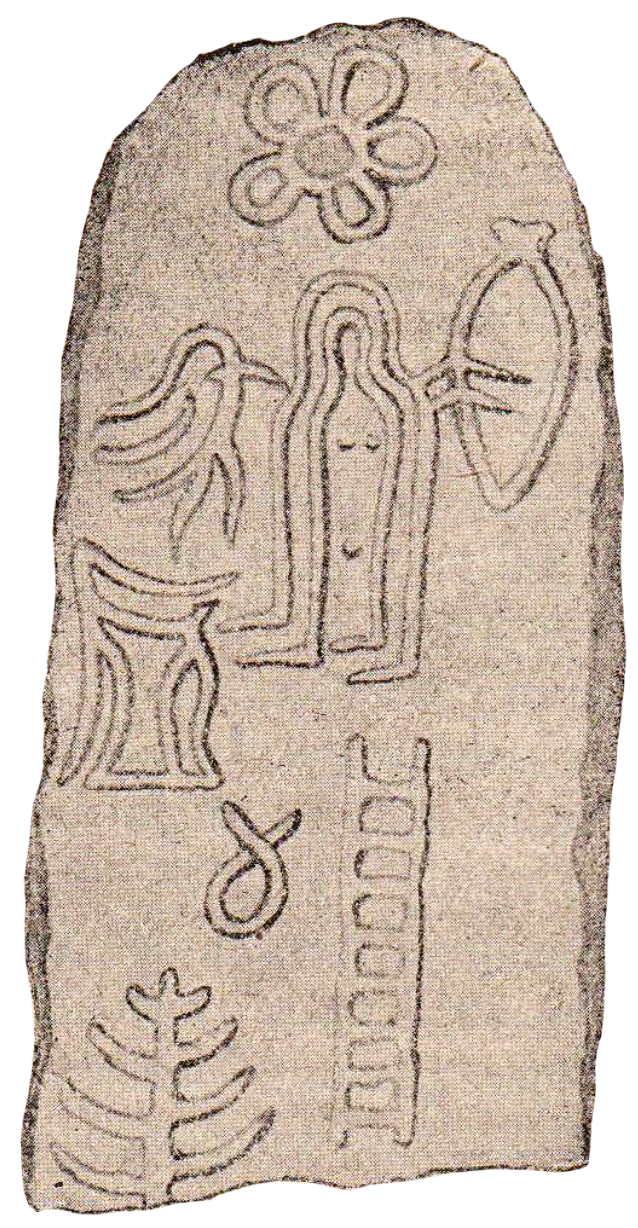

Fig. 3 : Stèle anépigraphe (d’après L. Carton, « Note sur des édicules ... », op. cit., n. 31, 345). 


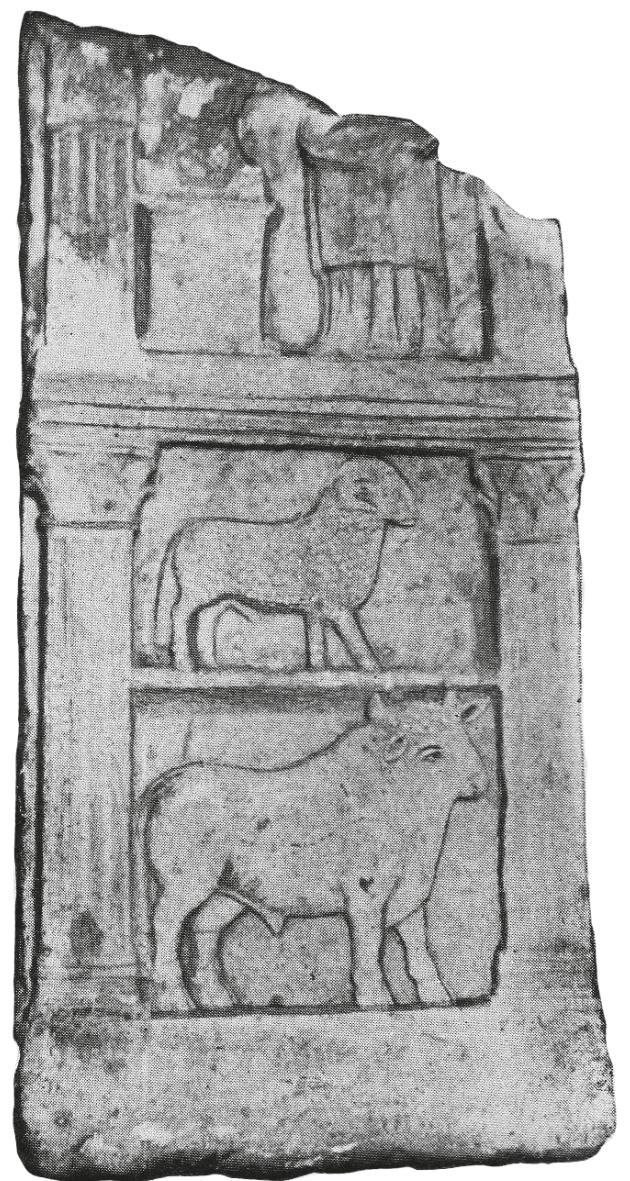

Fig. 4 : Stèle en marbre, El-Djem (d'après L. Carton, « Note sur quelques collections ...", op. cit., n. 32). 
Monógrafico | Louis Carton (1861-1924) et les religions d'Afrique

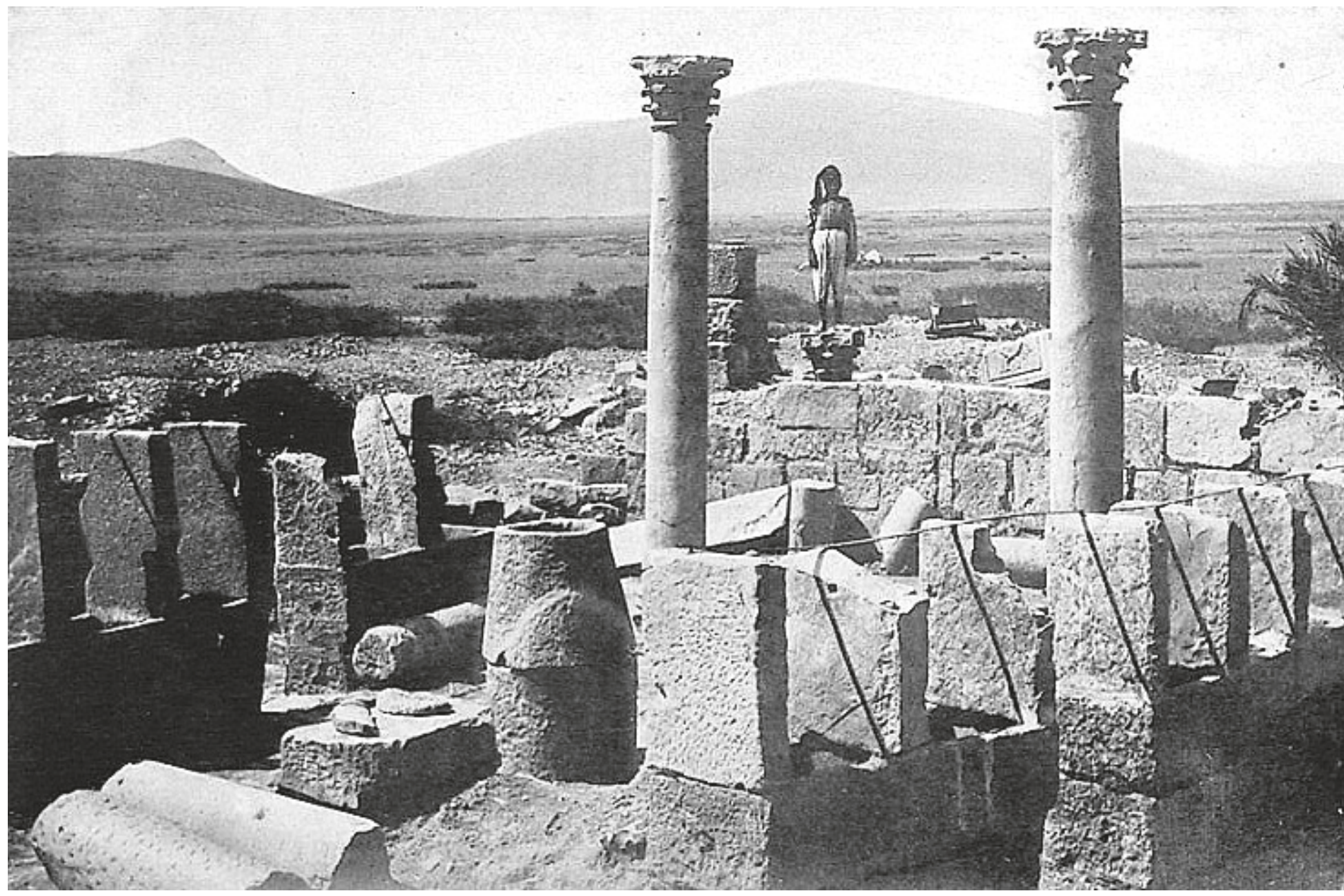

Fig. 5 : Les lignes d'auges de l'église du prêtre Alexander à Bulla Regia (d'après L. Carton, «Les lignes d'auges ... », op. cit., n. 36). 